\title{
Dropping acid: why is cystic fibrosis mucus abnormal?
}

\author{
Bruce K. Rubin ${ }^{1}$ and David J. Thornton ${ }^{2}$
}

Affiliations: 'Virginia Commonwealth University School of Medicine, Children's Hospital of Richmond at VCU, Richmond, VA, USA. ${ }^{2}$ Wellcome Trust Centre for Cell-Matrix Research and Lydia Becker Institute of Immunology and Inflammation, School of Biological Sciences, Faculty of Biology, Medicine and Health, University of Manchester, Manchester Academic Health Sciences Centre, Manchester, UK.

Correspondence: Bruce K. Rubin, Virginia Commonwealth University School of Medicine, Children's Hospital of Richmond at VCU, 1000 East Broad St, Richmond, VA 23298, USA. E-mail: bruce.rubinavcuhealth.com

@ERSpublications

In cystic fibrosis, abnormal mucus viscosity and mucociliary clearance are due to decreased water and more solids, and not to changes in mucus pH http://ow.ly/NvBS30myLwk

Cite this article as: Rubin BK, Thornton DJ. Dropping acid: why is cystic fibrosis mucus abnormal? Eur Respir J 2018; 52: 1802057 [https://doi.org/10.1183/13993003.02057-2018].

Cystic fibrosis (CF) is associated with the production of viscous and adherent mucus in the airway. Indeed, in some countries, CF is still called "mucoviscidosis". Mucus is a weak hydrogel exhibiting viscoelastic properties and these properties are important for normal function as they enable mucus to flow after secretion, to be effectively propelled by beating cilia (mucociliary clearance), and to provide a protective coating of the mucosal surfaces of our body that prevents dehydration and an exposure to external foreign substances and infectious agents. Mucus that is too thick or too thin can be difficult to transport by mucociliary clearance, and the production and accumulation of thick mucus in the airways eventually leads to mucostasis. The failure in function of mucus in airway clearance can lead to longer resident times of inhaled particulates including infectious agents, which results in chronic infection in the airway, lung damage and risk of death. Mucostasis and its associated problems are pathognomic features of the CF airway, and thus understanding the causes of abnormal mucus properties in CF is key to helping ameliorate the relentless progression of pulmonary disease.

$\mathrm{CF}$ results from mutations in the CF transmembrane conductance regulator protein, which functions to regulate chloride, bicarbonate, and secondarily sodium and water transport across the epithelial membrane [1]. CF disease-causing mutations can dysregulate the fluid content and composition of the airway surface liquid, including the periciliary layer (PCL), leading to increased mucus gel viscosity, PCL collapse and defective airway clearance $[2,3]$ (figure 1 ). It is also known that $\mathrm{pH}$ affects mucus viscosity such that acidic environments $(\mathrm{pH}<4)$, like in the stomach, will increase mucus viscosity, potentially protecting the gastric epithelium from hydrogen ion permeation through mucus and acid damage [4, 5]. CF airway secretions have a lower $\mathrm{pH}$ than the slightly alkaline mucus in the normal airway ( $\mathrm{pH} \sim 6.9$ compared to $\sim 7.1$ in newborn CF and normal pigs, respectively) due in part to inflammation, and in part to impaired bicarbonate secretion $[6,7]$. These findings have led to the development of two predominant hypotheses to explain the viscous and adherent mucus in CF: either failure of ion and water transport, so-called dehydration at the airway surface $[8,9]$, or acidification of the airway surface milieu [7], both of which lead to mucus hyperconcentration and increased viscoelasticity.

The effect of $\mathrm{pH}$ versus hydration has been a long-standing area of interest in understanding CF secretions. In a series of elegant studies published in this issue of the European Respiratory Journal, HiLL 
a) Health (functional MCC) Mucus transport $\rightarrow$

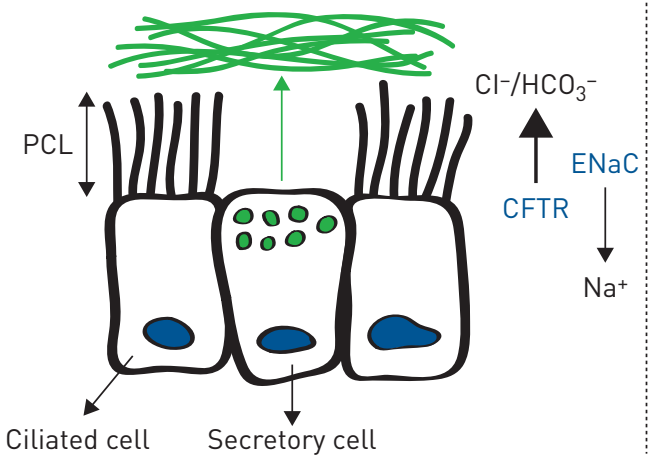

b)

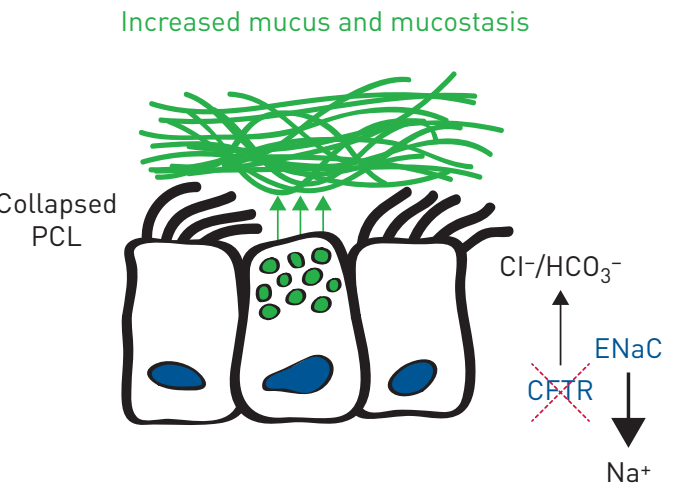

FIGURE 1 Cartoon showing the consequences of increased mucus concentration on mucociliary clearance (MCC) in the cystic fibrosis (CF) lung. a) Health (functional MCC); b) CF (dysfunctional MCC). PCL: periciliary layer; CFTR: CF transmembrane conductance regulator; ENaC: epithelial sodium channel.

et al. [10] demonstrated that the viscoelastic properties of mucus collected from cultures of normal human bronchial epithelium, as well as CF epithelium and CF sputum, were affected to a much greater extent by changes in solids concentration (and inversely changes in water composition) than alterations in $\mathrm{pH}$ across a physiological range observed in the airway. Microrheology describes how extremely small particles, like drugs, can penetrate the mucus layer and this was measured as the ability for a micrometre-sized rheologic probe to permeate the mucus gel mesh. The microrheology of mucus was more influenced by $\mathrm{pH}$ than the macroscopic rheological properties (macrorheology) or the viscoelastic response of the gel itself. In marked contrast, both micro- and macrorheology exhibited a far greater response to changes in mucus concentration (hydration) than to $\mathrm{pH}$. Furthermore, these increases in viscoelasticity were closely associated with a decreased mucociliary clearance which could then be reversed by the addition of a mucolytic agent but importantly, not by an alkalinisation of the secretions.

These data support that the biophysical alterations in mucus and sputum rheology seen in CF are dominated by changes in the solids concentration (hydration) of the mucus layer, and not by alterations in $\mathrm{pH}$. Nevertheless, the acidic milieu has been shown to have adverse effects on airway defence and inflammation, and potentially on the effectiveness of inhaled medications [6]. These alterations in antimicrobial defence probably contribute to the chronic infection seen in $\mathrm{CF}$, which leads to bronchiectasis and respiratory morbidity. It may be that the most effective therapies for the abnormalities in mucus at the airway surface will include the judicious use of mucolytics together with airway alkalisation. Clinical studies involving these therapeutic combinations will truly be the acid test to determine the most effective approach to treating CF lung disease.

\section{Conflict of interest: None declared.}

\section{References}

1 Gadsby DC, Vergani P, Csanády L. The ABC protein turned chloride channel whose failure causes cystic fibrosis. Nature 2006; 440: 477-483.

2 Sheppard DN, Welsh MJ. Structure and function of the CFTR chloride channel. Physiol Rev 1999; 79: S23-S45.

3 Button B, Cai L-H, Ehre C, et al. Periciliary brush promotes the lung health by separating the mucus layer from airway epithelia. Science 2012; 337: 937-941.

4 Celli JP, Turner BS, Afdhal NH, et al. Rheology of gastric mucin exhibits a pH-dependent sol-gel transition. Biomacromolecules 2007; 8: 1580-1586.

5 Bhaskar KR, Garik P, Turner BS, et al. Viscous fingering of $\mathrm{HCl}$ through gastric mucin. Nature 1992; 360: 458-461.

6 Pezzulo AA, Tang XX, Hoegger MJ, et al. Reduced airway surface $\mathrm{pH}$ impairs bacterial killing in the porcine cystic fibrosis lung. Nature 2012; 487: 109-113.

7 Tang XX, Ostedgaard LS, Hoegger MJ, et al. Acidic pH increases airway surface liquid viscosity in cystic fibrosis J Clin Invest 2016; 126: 879-891.

8 Matsui $\mathrm{H}$, Grubb BR, Tarran R, et al. Evidence for periciliary liquid layer depletion, not abnormal ion composition, in the pathogenesis of cystic fibrosis airways disease. Cell 1998; 95: 1005-1015.

9 Donaldson SH, Boucher RC. Sodium channels and cystic fibrosis. Chest 2007; 132: 1631-1636.

10 Hill DB, Long RF, Kissner WJ, et al. Pathological mucus and impaired mucus clearance in cystic fibrosis patients result from increased concentration, not altered pH. Eur Respir J 2018; 52: 1801297. 